\title{
Fitted Numerical Scheme for Second-Order Singularly Perturbed Differential-Difference Equations with Mixed Shifts
}

\author{
Meku Ayalew $\mathbb{D}^{1},{ }^{1}$ Gashu Gadisa Kiltu $\mathbb{D}^{\circ},{ }^{2}$ and Gemechis File Duressa ${ }^{1}{ }^{1}$ \\ ${ }^{1}$ Department of Mathematics, Jimma University, P.O. Box 378, Jimma, Ethiopia \\ ${ }^{2}$ Department of Mathematics, Madda Walabu University, P.O. Box 247, Bale Robe, Ethiopia \\ Correspondence should be addressed to Gashu Gadisa Kiltu; gashugadisa4@gmail.com
}

Received 6 April 2021; Revised 28 May 2021; Accepted 14 June 2021; Published 1 July 2021

Academic Editor: Chun Gang Zhu

Copyright (C) 2021 Meku Ayalew et al. This is an open access article distributed under the Creative Commons Attribution License, which permits unrestricted use, distribution, and reproduction in any medium, provided the original work is properly cited.

\begin{abstract}
This paper presents the study of singularly perturbed differential-difference equations of delay and advance parameters. The proposed numerical scheme is a fitted fourth-order finite difference approximation for the singularly perturbed differential equations at the nodal points and obtained a tridiagonal scheme. This is significant because the proposed method is applicable for the perturbation parameter which is less than the mesh size, where most numerical methods fail to give good results. Moreover, the work can also help to introduce the technique of establishing and making analysis for the stability and convergence of the proposed numerical method, which is the crucial part of the numerical analysis. Maximum absolute errors range from $10^{-03}$ up to $10^{-10}$, and computational rate of convergence for different values of perturbation parameter, delay and advance parameters, and mesh sizes are tabulated for the considered numerical examples. Concisely, the present method is stable and convergent and gives more accurate results than some existing numerical methods reported in the literature.
\end{abstract}

\section{Introduction}

Singularly perturbed differential-difference equations (SPDDEs) occur frequently in the mathematical modeling of various physical and biological phenomena, for example, control theory, viscous elasticity, and population dynamics [1]. Recently, many researchers have started developing different numerical methods for solving differential equations. Reference [2] investigated that the nonlinear thermal radiation and dissipation with the Darcy-Forchheimer equation in the porous medium analysis by using the fifth-order Runge-Kutta method, [3] also discussed the cross-fluid flow containing gyrotactic microorganisms and nanoparticles on a horizontal and three-dimensional cylinder by using the Runge-Kutta Fehlberg fifth-order technique, [4] studied the three-dimensional convective heat transfer of magnetohydrodynamics nanofluid flow through a rotating cone by using the fifth-order Runge-Kutta method. Reference [5] discussed that the heat transfer hybrid nanofluid contains 1-butanol as the base fluid and $\mathrm{MoS}_{2}-\mathrm{Fe}_{3} \mathrm{O}_{4}$ hybrid nanoparticles by using the finite element method. In these papers, the influence of various parameters on velocity profile and temperature has been investigated. Reference [6] presented a finite difference numerical method for solving singularly perturbed delay differential equations, [7] introduced a Galerkin method for solving this problem, [8] also introduced a fitted tension spline method for solving such problem, [9] presented a fitted second-order numerical method for singularly perturbed problems, [10-14] also developed some numerical methods of different orders for solving singularly perturbed delay differential equations, and so on. However, the issue of convergence and accuracy still needs attention and improvement. In this paper, we present a stable, convergent, and more accurate exponentially fitted fourth-order numerical scheme for solving SPDDEs and investigate the influence of delay and advance parameters on the solution profile. 


\section{Statement of the Problem of the Exponentially Fitted Method}

Consider the governing equation $[7,14,15]$ :

$$
\begin{aligned}
\varepsilon y^{\prime \prime}(x) & +\phi(x) y^{\prime}(x)+\psi(x) y(x-\delta)+\varphi(x) y(x) \\
& +\vartheta(x) y(x+\eta)=r(x), x \in[0,1]
\end{aligned}
$$

subject to the boundary conditions,

$$
y(x)=\alpha(x),-\delta \leq x \leq 0, y(x)=\beta(x), 1 \leq x \leq 1+\eta
$$

where $\varepsilon$ is a perturbation parameter $(0<\varepsilon<<1), \delta$ is a delay parameter, $\eta$ is the advance parameter with $0<\delta, \eta=o(\varepsilon)$, and $\phi(x), \psi(x), \varphi(x), \vartheta(x), r(x), \alpha(x)$ and $\beta(x)$ are smooth functions on $(0,1)$. Depending on the sign of $\psi(x)+\varphi(x)$ $+\vartheta(x)$, different cases of boundary layers are reported in [12].

From the Taylor series expansion in the neighborhood of the point $x$, we obtain

$$
\begin{gathered}
y(x-\delta) \approx y(x)-\delta y^{\prime}(x)+o\left(\delta^{2}\right), \\
y(x+\eta) \approx y(x)+\eta y^{\prime}(x)+o\left(\eta^{2}\right) .
\end{gathered}
$$

Replacing Equations (3) and (4) into Equation (1) gives an asymptotically equivalent SP two-point boundary value problem:

$$
L y(x) \equiv \varepsilon y^{\prime \prime}(x)+p(x) y^{\prime}(x)+q(x) y(x)=r(x),
$$

under the boundary conditions,

$$
y(0)=\alpha_{0}, \quad y(1)=\beta_{0},
$$

where $p(x)=\phi(x)-\delta \psi(x)+\eta \vartheta(x)$ and $q(x)=\psi(x)+\varphi(x)$ $+\vartheta(x)$.

The transformation from Equation (1) with Equation (5) is accepted, because the conditions $0<\delta, \eta<<1$ are sufficiently small [16]

Using the uniform mesh technique over the domain, we have $x_{i}=x_{0}+i h, i=0,1, \cdots, N$.

By using the Taylor series expansion, we obtain

$$
\begin{gathered}
y_{i+1}=y_{i}+h y_{i}^{\prime}+\frac{h^{2}}{2 !} y_{i}^{\prime \prime}+\frac{h^{3}}{3 !} y_{i}^{(3)}+\frac{h^{4}}{4 !} y_{i}^{(4)}+o\left(h^{5}\right), \\
y_{i-1}=y_{1}-h y_{i}^{\prime}+\frac{h^{2}}{2 !} y_{i}^{\prime \prime}-\frac{h^{3}}{3 !} y_{i}^{(3)}+\frac{h^{4}}{4 !} y_{i}^{(4)}+o\left(h^{5}\right) .
\end{gathered}
$$

Subtracting Equation (8) from Equation (7) gives the approximation $\delta_{c}^{1} y_{i}$, for the first derivative of $y_{i}$ as

$$
\delta_{c}^{1} y_{i}=\frac{y_{i+1}-y_{i-1}}{2 h}+T_{1} \text {, }
$$

where $T_{1}=-\left(h^{2} / 6\right) y_{i}^{(3)}$.
Similarly, adding Equations (7) and (8) provides the approximation $\delta_{c}^{2} y_{i}$, for the second derivative of $y_{i}$ as

$$
\delta_{c}^{2} y_{i}=\frac{y_{i+1}-2 y_{i}+y_{i-1}}{h^{2}}+T_{2}
$$

where $T_{2}=-\left(h^{2} / 12\right) y_{i}^{(4)}$.

Substituting Equations (7) and (8) into Equation (9) yields

$$
\delta_{c}^{1} y_{i}=y_{i}^{\prime}+\frac{h^{2}}{6} y_{i}^{(3)}+T_{3}
$$

where $T_{3}=\left(h^{4} / 120\right) y_{i}^{(5)}+T_{1}=\left(h^{4} / 120\right) y_{i}^{(5)}-\left(h^{2} / 6\right) y_{i}^{(3)}$.

Again, substituting Equations (7) and (8) into Equation (10) gives

$$
\delta_{c}^{2} y_{i}=y_{i}^{\prime \prime}+\frac{h^{2}}{12} y_{i}^{(4)}+T_{4},
$$

where $T_{4}=\left(h^{4} / 360\right) y_{i}^{(6)}-\left(h^{2} / 12\right) y_{i}^{(4)}$.

Applying $\delta_{c}^{2}$ to $y_{i}^{\prime}$ in Equation (9), we get

$$
y_{i}^{(3)}=\delta_{c}^{2} y_{i}^{\prime}-T_{1}^{(2)} \text {. }
$$

Using Equation (13) into Equation (11), we obtain

$$
\delta_{c}^{1} y_{i}=y_{i}^{\prime}+\frac{h^{2}}{6} \delta_{c}^{2} y_{i}^{\prime}+T_{5}
$$

where $T_{5}=\left(13 h^{4} / 360\right) y_{i}^{(5)}-\left(h^{2} / 6\right) y_{i}^{(3)}$.

Applying $\delta_{c}^{2}$ to $y_{i}^{\prime \prime}$ in Equation (10), we get a four-order finite difference scheme for Equation (5):

$$
y_{i}^{(4)}=\delta_{c}^{2} y_{i}^{\prime \prime}-T_{2}^{(2)}
$$

Substituting Equation (15) into Equation (12), we get

$$
\delta_{c}^{2} y_{i}=y_{i}^{\prime \prime}+\frac{h^{2}}{12} \delta_{c}^{2} y_{i}^{\prime \prime}+T_{6}
$$

where $T_{6}=\left(7 h^{4} / 720\right) y_{i}^{(6)}-\left(h^{2} / 12\right) y_{i}^{(4)}$.

From Equations (14) and (16), we get

$$
y_{i}^{\prime}=\frac{\delta_{c}^{1} y_{i}-T_{5}}{1+\left(h^{2} / 6\right) \delta_{c}^{2}}, y_{i}^{\prime \prime}=\frac{\delta_{c}^{2} y_{i}-T_{6}}{1+\left(h^{2} / 12\right) \delta_{c}^{2}} .
$$

After evaluating Equation (5) at nodal point $x_{i}$ and using Equation (17), we obtain

$$
\varepsilon\left(\frac{\delta_{c}^{2} y_{i}-T_{6}}{1+\left(h^{2} / 12\right) \delta_{c}^{2}}\right)+p_{i}\left(\frac{\delta_{c}^{1} y_{i}-T_{5}}{1+\left(h^{2} / 6\right) \delta_{c}^{2}}\right)+q_{i} y_{i}=r_{i} .
$$


Simplifying Equation (18), we have

$$
\begin{aligned}
\varepsilon \delta_{c}^{2} y_{i} & +\varepsilon \frac{h^{2}}{6} \delta_{c}^{4} y_{i}-\varepsilon\left(1+\frac{h^{2}}{6} \delta_{c}^{2}\right) T_{6}+p_{i} \delta_{c}^{1} y_{i} \\
& +\frac{h^{2} p_{i}}{12} \delta_{c}^{3} y_{i}+q_{i} y_{i}+\frac{h^{2} q_{i}}{4} \delta_{c}^{2} y_{i}+\frac{h^{4} q_{i}}{72} \delta_{c}^{4} y_{i} \\
= & r_{i}\left(1+\frac{h^{2}}{4} \delta_{c}^{2}+\frac{h^{4}}{72} \delta_{c}^{4}\right)+p_{i} T_{5}\left(1+\frac{h^{2}}{12} \delta_{c}^{2}\right) .
\end{aligned}
$$

Rearranging Equation (5) and successively differentiating, evaluating at $x_{i}$, and substituting into Equation (19), we get

$$
\begin{aligned}
(\varepsilon- & \left.\frac{h^{2}}{6}\left(2 p_{i}^{\prime}\right)+\frac{h^{2} q_{i}}{12}+\frac{h^{2} p_{i}^{2}}{12 \varepsilon}-\frac{h^{4} q_{i}}{72 \varepsilon}\left(2 p_{i}^{\prime}+q_{i}-\frac{p_{i}^{2}}{\varepsilon}\right)\right) \delta_{c}^{2} y_{i} \\
& -\varepsilon\left(1+\frac{h^{2}}{6} \delta_{c}^{2}\right) T_{6}+\left\{-\frac{h^{2} p_{i}}{12 \varepsilon}\left(p_{i}^{\prime}+q_{i}\right)+\frac{h^{4} q_{i}}{72 \varepsilon}\right. \\
& \left.\cdot\left(\frac{p_{i}\left(p_{i}^{\prime}+q_{i}\right)}{\varepsilon}-p_{i}^{\prime \prime}-2 q_{i}^{\prime}\right)+\frac{h^{2}}{6}\left(\frac{p_{i}\left(p_{i}^{\prime}+q_{i}\right)}{\varepsilon}-p_{i}^{\prime \prime}-2 q_{i}^{\prime}\right)+p_{i}\right\} \delta_{c}^{1} y_{i} \\
& +\left\{\frac{h^{4} q_{i}}{72 \varepsilon}\left(\frac{p_{i} q_{i}^{\prime}}{\varepsilon}-q_{i}^{\prime \prime}\right)-\frac{h^{2} p_{i} q_{i}^{\prime}}{12 \varepsilon}+q_{i}+\frac{h^{2}}{6}\left(\frac{p_{i} q_{i}^{\prime}}{\varepsilon}-q_{i}^{\prime \prime}\right)\right\} y_{i} \\
= & r_{i}+\frac{h^{2}}{4} \delta_{c}^{2} r_{i}+\frac{h^{4}}{72} \delta_{c}^{2} r_{i}^{\prime \prime}+p_{i} T_{5}\left(1+\frac{h^{2}}{12} \delta_{c}^{2}\right)-\frac{h^{2}}{6} r_{i}^{\prime \prime} \\
& +\frac{h^{2} p_{i}}{12 \varepsilon} r_{i}^{\prime}-\frac{h^{4} q_{i}}{72 \varepsilon} r_{i}^{\prime \prime}-\frac{h^{4} p_{i} q_{i}}{72 \varepsilon^{2}} r_{i}^{\prime} .
\end{aligned}
$$

Introducing the fitting factor $(\sigma)$ into Equation (20), we have

$$
\begin{aligned}
\sigma \varepsilon\{ & \left(1-\frac{h^{2}}{6 \varepsilon}\left(2 p_{i}^{\prime}\right)+\frac{h^{2} q_{i}}{12 \varepsilon}+\frac{h^{2} p_{i}^{2}}{12 \varepsilon^{2}}-\frac{h^{4} q_{i}}{72 \varepsilon^{2}}\left(2 p_{i}^{\prime}+q_{i}-\frac{p_{i}^{2}}{\varepsilon}\right)\right) \delta_{c}^{2} y_{i} \\
& \left.-\left(1+\frac{h^{2}}{6} \delta_{c}^{2}\right) T_{6}\right\}+\left\{-\frac{h^{2} p_{i}}{12 \varepsilon}\left(p_{i}^{\prime}+q_{i}\right)+\frac{h^{4} q_{i}}{72 \varepsilon}\right. \\
& \left.\cdot\left(\frac{p_{i}\left(p_{i}^{\prime}+q_{i}\right)}{\varepsilon}-p_{i}^{\prime \prime}-2 q_{i}^{\prime}\right)+\frac{h^{2}}{6}\left(\frac{p_{i}\left(p_{i}^{\prime}+q_{i}\right)}{\varepsilon}-p_{i}^{\prime \prime}-2 q_{i}^{\prime}\right)+p_{i}\right\} \delta_{c}^{1} y_{i} \\
& +\left\{\frac{h^{4} q_{i}}{72 \varepsilon}\left(\frac{p_{i} q_{i}^{\prime}}{\varepsilon}-q_{i}^{\prime \prime}\right)-\frac{h^{2} p_{i} q_{i}^{\prime}}{12 \varepsilon}+q_{i}+\frac{h^{2}}{6}\left(\frac{p_{i} q_{i}^{\prime}}{\varepsilon}-q_{i}^{\prime \prime}\right)\right\} y_{i} \\
= & r_{i}+\frac{h^{2}}{4} \delta_{c}^{2} r_{i}+\frac{h^{4}}{72} \delta_{c}^{2} r_{i}^{\prime \prime}+p_{i} T_{5}\left(1+\frac{h^{2}}{12} \delta_{c}^{2}\right) \\
& -\frac{h^{2}}{6} r_{i}^{\prime \prime}+\frac{h^{2} p_{i}}{12 \varepsilon} r_{i}^{\prime}-\frac{h^{4} q_{i}}{72 \varepsilon} r_{i}^{\prime \prime}-\frac{h^{4} p_{i} q_{i}}{72 \varepsilon^{2}} r_{i}^{\prime} .
\end{aligned}
$$

Using the central difference approximation for $\delta_{c}^{2} y_{i}$ and $\delta_{c}^{1} y_{i}$, multiplying both sides of Equation (21) by $h$, and evaluating the limit as $h \longrightarrow 0$, we obtain $\frac{\sigma}{12 \rho}\left(12+\rho^{2} p_{i}^{2}\right) \lim _{h \longrightarrow 0}\left\{y_{i-1}-2 y_{i}+y_{i+1}\right\}+\frac{p_{i}}{2} \lim _{h \longrightarrow 0}\left(y_{i+1}-y_{i-1}\right)=0$,

where $\rho=(h / \varepsilon)$.

From the theory of singular perturbations and O'Malley [17], we have two cases for $p(x)>0$ and $p(x)<0$.

Case 1. For $p(x)<0$ (right-end boundary layer), we have

$$
\begin{aligned}
\lim _{h \longrightarrow 0}\left(y_{i-1}-2 y_{i}+y_{i+1}\right)= & \left\{\left(\alpha_{0}-y_{0}(0)\right) e^{-p(0)(-1 / \varepsilon+i \rho)}\right. \\
& \left.\cdot\left(e^{p(0) \rho}+e^{-p(0) \rho}-2\right)\right\}, \\
\lim _{h \longrightarrow 0}\left(y_{i+1}-y_{i-1}\right)= & \left\{\left(\alpha_{0}-y_{0}(0)\right) e^{-p(0)(-1 / \varepsilon+i \rho)}\right. \\
& \left.\cdot\left(e^{-p(0) \rho}-e^{p(0) \rho}\right)\right\} .
\end{aligned}
$$

Thus, from Equation (22), we get

$$
\sigma(0)=\frac{6 \rho p(0)}{\left(12+\rho^{2} p^{2}(0)\right)} \operatorname{coth}\left(\frac{p(0) \rho}{2}\right) .
$$

Case 2. For $p(x)>0$ (left-end boundary layer), we have

$$
\begin{aligned}
\lim _{h \longrightarrow 0}\left(y_{i-1}-2 y_{i}+y_{i+1}\right)= & \left\{\left(\beta_{0}-y_{0}(1)\right) e^{-p(1)(-1 / \varepsilon+i \rho)}\right. \\
& \left.\cdot\left(e^{-p(1) \rho}+e^{p(1) \rho}-2\right)\right\},
\end{aligned}
$$

$$
\begin{aligned}
\lim _{h \longrightarrow 0}\left(y_{i+1}-y_{i-1}\right)= & \left\{\left(\beta_{0}-y_{0}(1)\right) e^{-p(1)(-1 / \varepsilon+i \rho)}\right. \\
& \left.\cdot\left(e^{-p(1) \rho}-e^{p(1) \rho}\right)\right\}
\end{aligned}
$$

Thus, from Equation (22), we get

$$
\sigma(1)=\frac{6 \rho p(1)}{\left(12+\rho^{2} p^{2}(1)\right)} \operatorname{coth}\left(\frac{p(1) \rho}{2}\right) .
$$

In general, for discretization, we take a variable fitting parameter as

$$
\sigma_{i}=\frac{6 \rho p_{i}}{\left(12+\rho^{2} p_{i}^{2}\right)} \operatorname{coth}\left(\frac{p_{i} \rho}{2}\right)
$$

Now using Equations (9) and (10) into Equation (21) for $\delta_{c}^{1} y_{i}$ and $\delta_{c}^{2} y_{i}$ and making use of $\delta_{c}^{2} r_{i}=\left(r_{i-1}-2 r_{i}+r_{i+1}\right) / h^{2}$ and $\delta_{c}^{2} r_{i}^{\prime \prime}=\left(r_{i-1}^{\prime \prime}-2 r_{i}^{\prime \prime}+r_{i+1}^{\prime \prime}\right) / h^{2}$, we obtain 


$$
\begin{aligned}
\left\{\sigma_{i}(\right. & \left.\frac{\varepsilon}{h^{2}}+\frac{q_{i}}{12}-\frac{1}{6}\left(2 p_{i}^{\prime}\right)+\frac{p_{i}^{2}}{12 \varepsilon}-\frac{h^{2} q_{i}}{72 \varepsilon}\left(2 p_{i}^{\prime}+q_{i}-\frac{p_{i}^{2}}{\varepsilon}\right)\right) \\
& -\frac{1}{2}\left(-\frac{h p_{i}}{12 \varepsilon}\left(p_{i}^{\prime}+q_{i}\right)+\frac{h^{3} q_{i}}{72 \varepsilon}\left(-p_{i}^{\prime \prime}-2 q_{i}^{\prime}+\frac{p_{i}\left(p_{i}^{\prime}+q_{i}\right)}{\varepsilon}\right)\right. \\
& \left.\left.+\frac{h}{6}\left(-p_{i}^{\prime \prime}-2 q_{i}^{\prime}+\frac{p_{i}\left(p_{i}^{\prime}+q_{i}\right)}{\varepsilon}\right)+\frac{p_{i}}{h}\right)\right\} y_{i-1} \\
& +\left\{-2 \sigma_{i}\left(\frac{\varepsilon}{h^{2}}-\frac{1}{6}\left(2 p_{i}^{\prime}\right)+\frac{q_{i}}{12}+\frac{p_{i}^{2}}{12 \varepsilon}-\frac{h^{2} q_{i}}{72 \varepsilon}\left(q_{i}+2 p_{i}^{\prime}-\frac{p_{i}^{2}}{\varepsilon}\right)\right)\right. \\
& \left.+\frac{h^{4} q_{i}}{72 \varepsilon}\left(-q_{i}^{\prime \prime}+\frac{p_{i} q_{i}^{\prime}}{\varepsilon}\right)-\frac{h^{2} p_{i} q_{i}^{\prime}}{12 \varepsilon}+q_{i}+\frac{h^{2}}{6}\left(\frac{p_{i} q_{i}^{\prime}}{\varepsilon}-q_{i}^{\prime \prime}\right)\right\} y_{i} \\
& +\left\{\sigma_{i}\left(\frac{\varepsilon}{h^{2}}-\frac{1}{6}\left(2 p_{i}^{\prime}\right)+\frac{q_{i}}{12}+\frac{p_{i}^{2}}{12 \varepsilon}-\frac{h^{2} q_{i}}{72 \varepsilon}\left(2 p_{i}^{\prime}+q_{i}-\frac{p_{i}^{2}}{\varepsilon}\right)\right)\right. \\
& +\frac{1}{2}\left(-\frac{h p_{i}}{12 \varepsilon}\left(p_{i}^{\prime}+q_{i}\right)+\frac{h^{3} q_{i}}{72 \varepsilon}\left(-p_{i}^{\prime \prime}-2 q_{i}^{\prime}+\frac{p_{i}\left(p_{i}^{\prime}+q_{i}\right)}{\varepsilon}\right)\right. \\
& \left.\left.+\frac{h}{6}\left(-p_{i}^{\prime \prime}-2 q_{i}^{\prime}+\frac{p_{i}\left(p_{i}^{\prime}+q_{i}\right)}{\varepsilon}\right)+\frac{p_{i}}{h}\right)\right\} y_{i+1} \\
= & r_{i}+\frac{1}{4}\left(r_{i-1}-2 r_{i}+r_{i+1}\right)+\frac{h^{2}}{72}\left(r_{i-1}^{\prime \prime}-2 r_{i}^{\prime \prime}+r_{i+1}^{\prime \prime}\right) \\
& -\frac{h^{2}}{6} r_{i}^{\prime \prime}+\frac{h^{2} p_{i}}{12 \varepsilon} r_{i}^{\prime}-\frac{h^{4} q_{i}}{72 \varepsilon} r_{i}^{\prime \prime}-\frac{h^{4} p_{i} q_{i}}{72 \varepsilon^{2}} r_{i}^{\prime}+T_{i},
\end{aligned}
$$

where $T_{i}=p_{i}\left(h^{4} / 45\right) y_{i}^{(5)}-\sigma_{i} \varepsilon\left(h^{4} / 240\right) y_{i}^{(6)}+O\left(h^{5}\right)$ is a local truncation error.

Simplifying Equation (24), we get a tridiagonal system:

$$
L^{N} \equiv A_{i} y_{i-1}-B_{i} y_{i}+C_{i} y_{i+1}=D_{i}, \quad \text { for } i=1,2, \cdots, N-1 \text {, }
$$

where

$$
\begin{aligned}
A_{i}= & \sigma_{i}\left\{\frac{\varepsilon}{h^{2}}+\frac{q_{i}}{12}-\frac{1}{6}\left(2 p_{i}^{\prime}\right)+\frac{p_{i}^{2}}{12 \varepsilon}-\frac{h^{2} q_{i}}{72 \varepsilon}\left(2 p_{i}^{\prime}+q_{i}-\frac{p_{i}^{2}}{\varepsilon}\right)\right\} \\
& -\frac{1}{2}\left\{-\frac{h p_{i}}{12 \varepsilon}\left(p_{i}^{\prime}+q_{i}\right)+\frac{h^{3} q_{i}}{72 \varepsilon}\left(\frac{p_{i}\left(p_{i}^{\prime}+q_{i}\right)}{\varepsilon}-p_{i}^{\prime \prime}-2 q_{i}^{\prime}\right)\right. \\
& \left.+\frac{h}{6}\left(\frac{p_{i}\left(p_{i}^{\prime}+q_{i}\right)}{\varepsilon}-p_{i}^{\prime \prime}-2 q_{i}^{\prime}\right)+\frac{p_{i}}{h}\right\}, \\
B_{i}=2 & \sigma_{i}\left\{\frac{\varepsilon}{h^{2}}-\frac{1}{6}\left(2 p_{i}^{\prime}\right)+\frac{q_{i}}{12}+\frac{p_{i}^{2}}{12 \varepsilon}-\frac{h^{2} q_{i}}{72 \varepsilon}\left(2 p_{i}^{\prime}+q_{i}-\frac{p_{i}^{2}}{\varepsilon}\right)\right\} \\
& -\frac{h^{4} q_{i}}{72 \varepsilon}\left(\frac{p_{i} q_{i}^{\prime}}{\varepsilon}-q_{i}^{\prime \prime}\right)+\frac{h^{2} p_{i} q_{i}^{\prime}}{12 \varepsilon}-q_{i}-\frac{h^{2}}{6}\left(\frac{p_{i} q_{i}^{\prime}}{\varepsilon}-q_{i}^{\prime \prime}\right),
\end{aligned}
$$

$$
\begin{aligned}
C_{i}= & \sigma_{i}\left\{\frac{\varepsilon}{h^{2}}-\frac{1}{6}\left(2 p_{i}^{\prime}\right)+\frac{q_{i}}{12}+\frac{p_{i}^{2}}{12 \varepsilon}-\frac{h^{2} q_{i}}{72 \varepsilon}\left(2 p_{i}^{\prime}+q_{i}-\frac{p_{i}^{2}}{\varepsilon}\right)\right\} \\
& +\frac{1}{2}\left\{-\frac{h p_{i}}{12 \varepsilon}\left(p_{i}^{\prime}+q_{i}\right)+\frac{h^{3} q_{i}}{72 \varepsilon}\left(\frac{p_{i}\left(p_{i}^{\prime}+q_{i}\right)}{\varepsilon}-p_{i}^{\prime \prime}-2 q_{i}^{\prime}\right)\right. \\
& \left.+\frac{h}{6}\left(\frac{p_{i}\left(p_{i}^{\prime}+q_{i}\right)}{\varepsilon}-p_{i}^{\prime \prime}-2 q_{i}^{\prime}\right)+\frac{p_{i}}{h}\right\}, \\
D_{i}= & r_{i}+\frac{1}{4}\left(r_{i-1}-2 r_{i}+r_{i+1}\right)+\frac{h^{2}}{72}\left(r_{i-1}^{\prime \prime}-2 r_{i}^{\prime \prime}+r_{i+1}^{\prime \prime}\right) \\
& -\frac{h^{2}}{6} r_{i}^{\prime \prime}+\frac{h^{2} p_{i}}{12 \varepsilon} r_{i}^{\prime}-\frac{h^{4} q_{i}}{72 \varepsilon} r_{i}^{\prime \prime}-\frac{h^{4} p_{i} q_{i}}{72 \varepsilon^{2}} r_{i}^{\prime} .
\end{aligned}
$$

We have used a discrete invariant imbedding algorithm to solve Equation (25).

\section{Stability Analysis}

The continuous minimum principle, continuous maximum principle, and stability of the solution of Equations (5) and (6) are presented in [13]. We present the stability of the scheme in Equation (25) for both cases.

Case 1. When $q(x)<0$, i.e., $\psi(x)+\varphi(x)+9(x)<0$, for $x \in($ $0,1)$.

Lemma 1 (discrete minimum principle). If $w_{i}$ is any mesh function such that $w_{o} \geq 0$ and $L^{N} w_{i} \leq 0$, then $w_{i} \geq 0$ for all $x_{i} \in(0,1)$.

Proof. Suppose $\exists k \in \mathbb{Z}^{+}$such that $w_{k}<0$ and $w_{k}=\min _{0 \leq i \leq N} w_{i}$.

Then, from Equation (25), we have

$$
\begin{aligned}
L^{N} w_{k} \equiv & A_{k} w_{k-1}-B_{k} w_{k}+C_{k} w_{k+1} \\
= & \left\{\left(\frac{\sigma_{k} \varepsilon}{h^{2}}+\frac{p_{k}^{2}}{12 \varepsilon}+\frac{q_{k}}{12}-\frac{p_{k}^{\prime}}{3}\right)+\frac{h^{2} q_{k}}{72 \varepsilon}\left(\frac{p_{k}^{2}}{\varepsilon}-2 p_{k}^{\prime}-q_{k}\right)\right\} \\
& \cdot\left(w_{k-1}-w_{k}\right)+\left\{\left(\frac{\sigma_{k} \varepsilon}{h^{2}}+\frac{p_{k}^{2}}{12 \varepsilon}+\frac{q_{k}}{12}-\frac{p_{k}^{\prime}}{3}\right)\right. \\
& \left.+\frac{h^{2} q_{k}}{72 \varepsilon}\left(\frac{p_{k}^{2}}{\varepsilon}-2 p_{k}^{\prime}-q_{k}\right)\right\}\left(w_{k+1}-w_{k}\right) \\
& +\left\{\frac{p_{k}}{h}+\frac{h p_{k}\left(p_{k}^{\prime}+q_{k}\right)}{12 \varepsilon}-\frac{h p_{k}^{\prime \prime}}{6}-\frac{h q_{k}^{\prime}}{3}+\frac{h^{3} q_{k}}{72 \varepsilon}\right. \\
& \left.\cdot\left(\frac{p_{k}\left(p_{k}^{\prime}+q_{k}\right)}{\varepsilon}-p_{k}^{\prime \prime}-2 q_{k}^{\prime}\right)\right\}\left(w_{k+1}-w_{k-1}\right)+F_{k} w_{k},
\end{aligned}
$$

where $F_{k}=\left(h^{2} / 6\right)\left(p_{k} q_{k}^{\prime} / \varepsilon-q_{k}^{\prime \prime}\right)-h^{2} p_{k} q_{k}^{\prime} / 12 \varepsilon+q_{k}+\left(h^{4} q_{k} / 72 \varepsilon\right.$ )$\left(p_{k} q_{k}^{\prime} / \varepsilon-q_{k}^{\prime \prime}\right)$. 


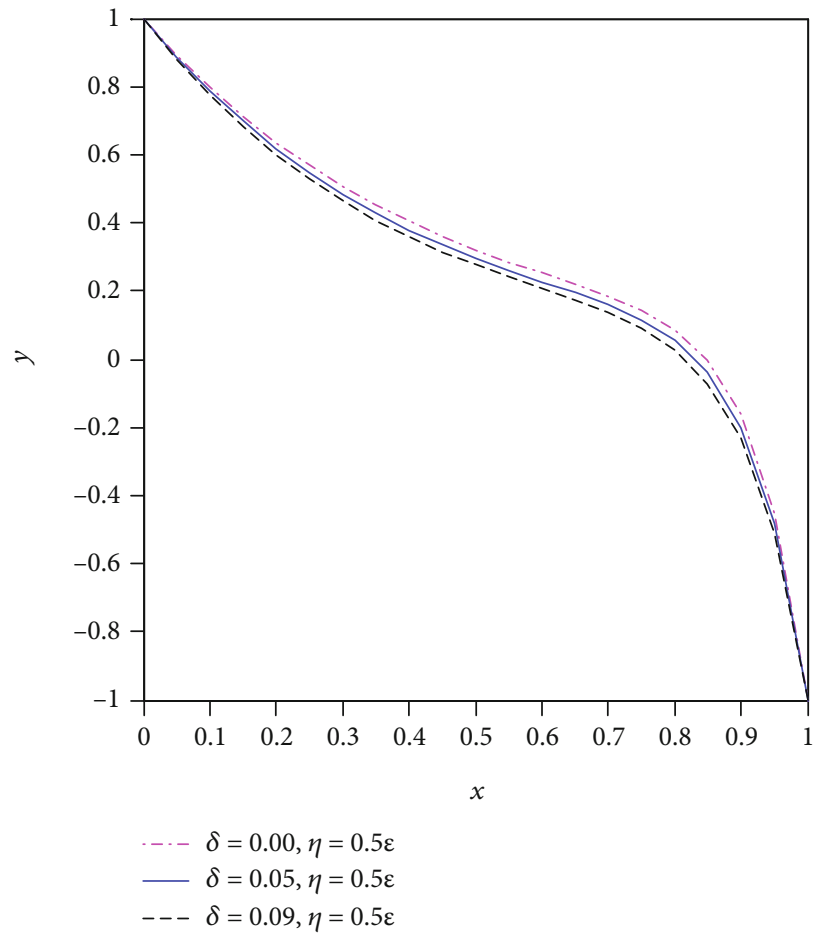

(a)

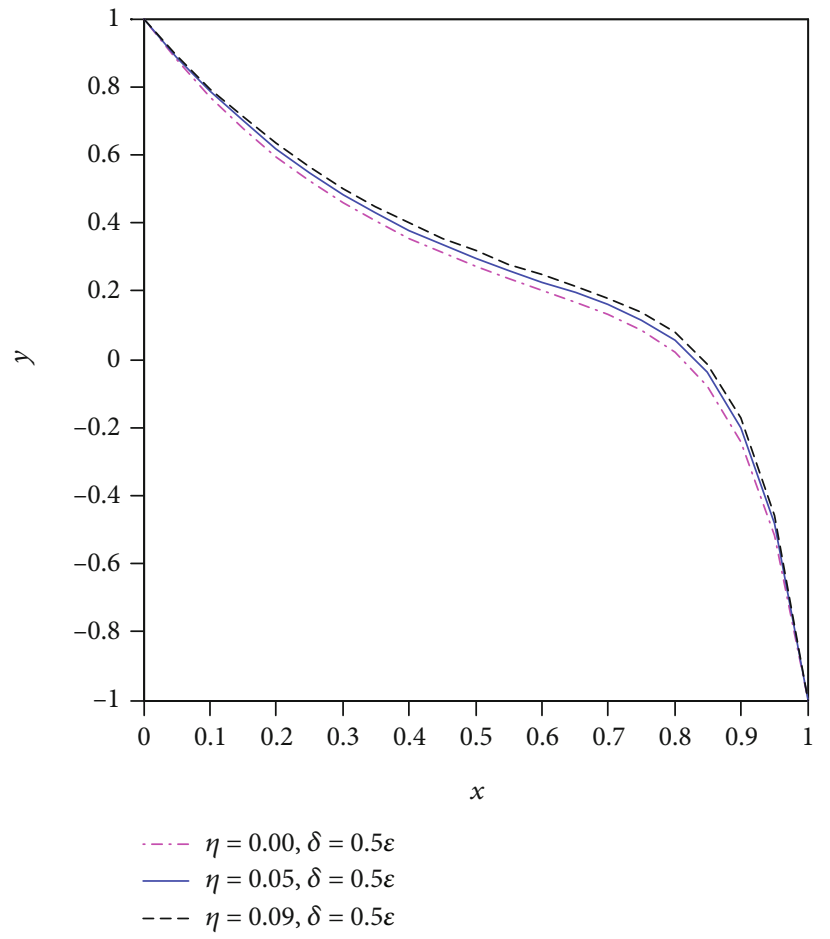

(b)

FIgURE 1: Numerical solution of Example 5 for $\varepsilon=0.1$ and $N=20$.

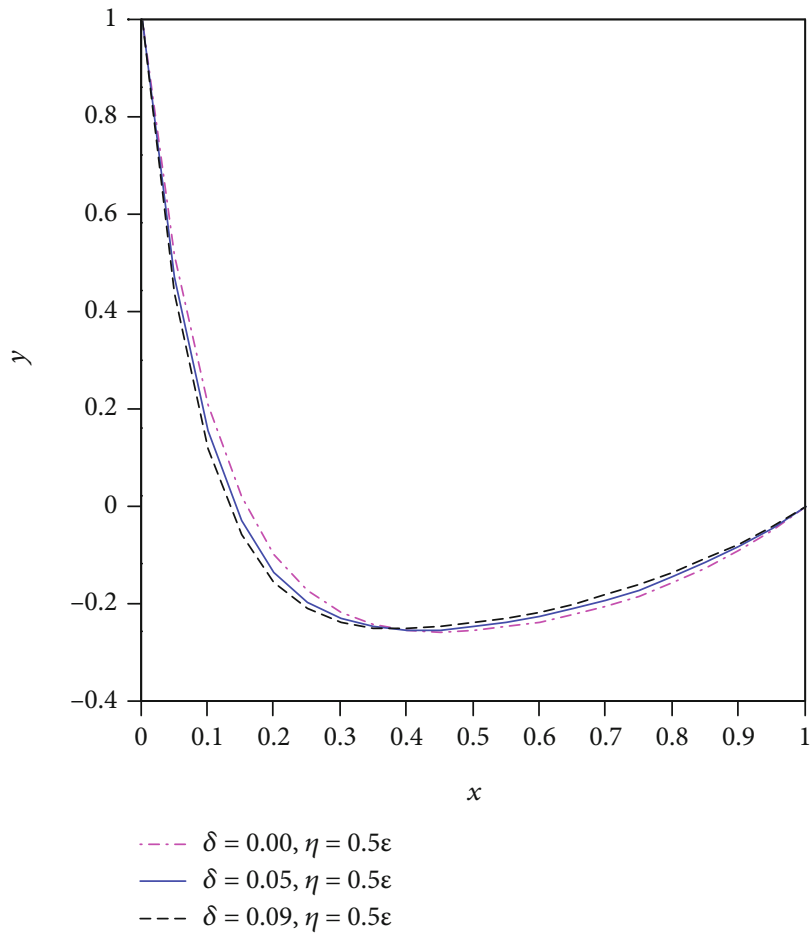

(a)

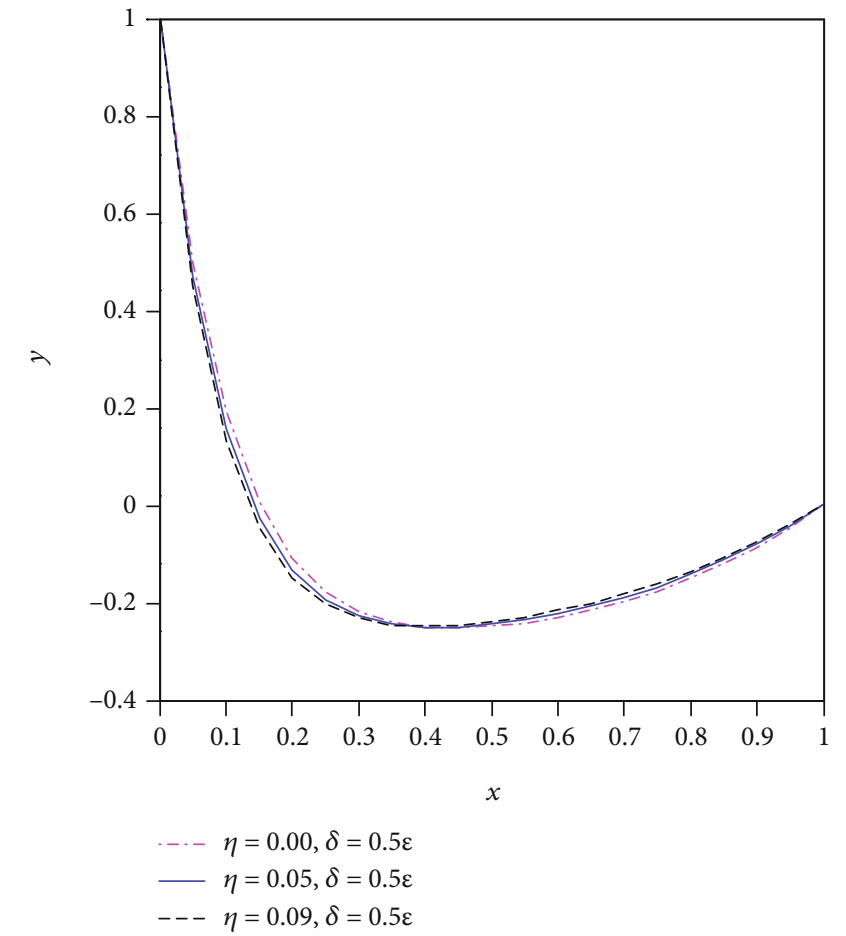

(b)

Figure 2: Numerical solution of Example 6 for $\varepsilon=0.1$ and $N=20$. 


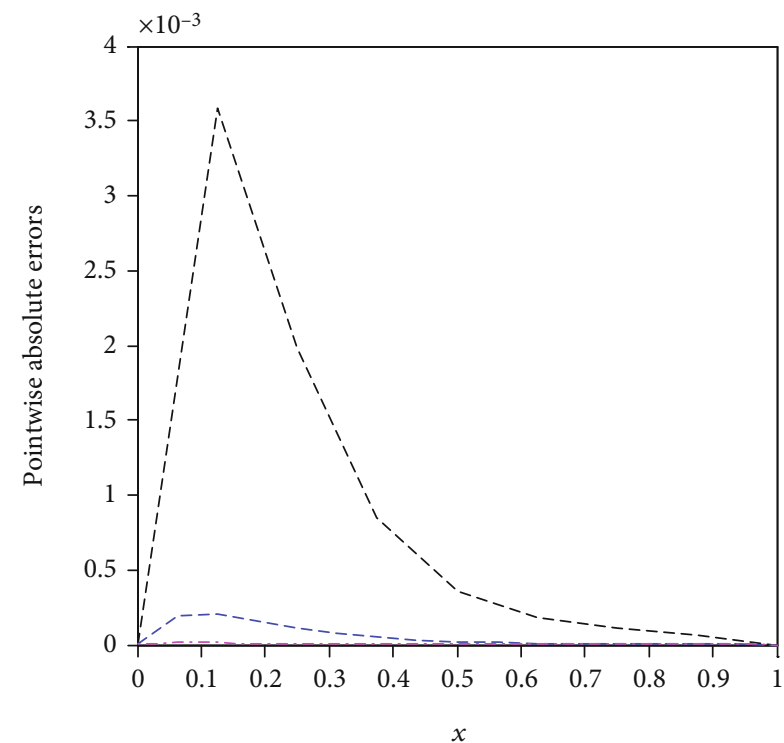

(a)

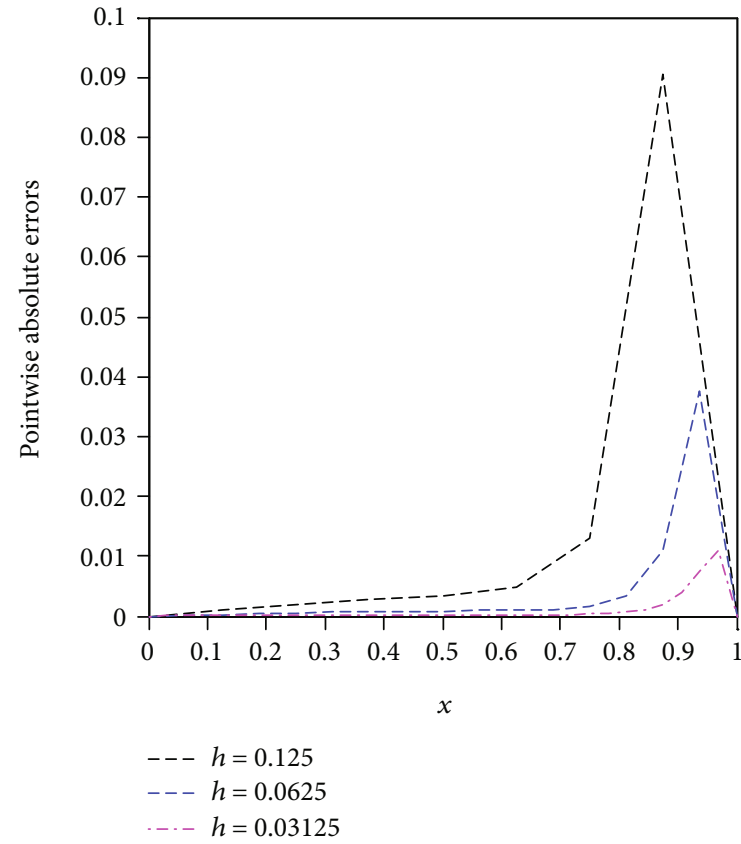

(b)

Figure 3: Pointwise absolute errors of Examples 6 and 7, respectively, for $\varepsilon=0.1$ and $\delta, \eta=0.5 \varepsilon$.

TABLE 1: Rate of convergence for $\varepsilon=0.1, \eta=0.5 \varepsilon$.

\begin{tabular}{ccccc}
\hline$\frac{\delta}{N}$ & 16 & 32 & 64 & 128 \\
\hline $\begin{array}{c}\text { Example } 5 \\
0.00\end{array}$ & 4.0655 & 4.0164 & 4.0041 & 4.0010 \\
0.05 & 4.0566 & 4.0144 & 4.0036 & 4.0009 \\
0.09 & 4.0500 & 4.0126 & 4.0031 & 4.0008 \\
Example 6 & & & & \\
0.00 & 4.0256 & 4.0064 & 4.0016 & 4.0004 \\
0.05 & 4.0349 & 4.0087 & 4.0022 & 4.0005 \\
0.09 & 4.0435 & 4.0108 & 4.0027 & 4.0007 \\
\hline
\end{tabular}

For sufficiently small $h$ (i.e., $h \longrightarrow 0$ ) and for suitable values of $p_{k}$, we obtain $L^{N} w_{k}>0$. Since, $w_{k}<0$ (by assumption), $\varepsilon, \sigma_{k}>0$ and $F_{k} \longrightarrow q_{k}<0$..

However, this is a contradiction. Hence, $w_{i} \geq 0$ for all $x_{i}$ $\in(0,1)$.

Theorem 2. The operator $L^{N}$ in Equation (25) is stable for $\psi$ $(x)+\varphi(x)+\vartheta(x)<0$ if $w_{i}$ is any mesh function, then $\left|w_{i}\right| \leq$ $\lambda \max \left\{\left|w_{0}\right|, \max _{x_{i} \in(0,1)}\left|L w_{i}\right|\right\}$, for some constant $\lambda \geq 1$.

Proof. (see [13]). This proves the stability of the scheme for the case of boundary layer behaviour.

Case 2. When $q(x)>0$, i.e., $\psi(x)+\varphi(x)+\vartheta(x)>0$, for $x \in($ $0,1)$.
Lemma 3 (discrete maximum principle). If $w_{i}$ is any mesh function such that $w_{0} \geq 0$ and $L^{N} w_{i} \geq 0$, then $w_{i} \geq 0$ for all $x_{i} \in(0,1)$.

Proof. Suppose $\exists k \in \mathbb{Z}^{+}$such that $w_{k}<0$ and $w_{k}=\max _{0 \leq i \leq N} w_{i}$.

Then, from Equation (25), we have

$$
\begin{aligned}
L^{N} w_{k} \equiv & A_{k} w_{k-1}-B_{k} w_{k}+C_{k} w_{k+1} \\
= & \left\{\left(\frac{\sigma_{k} \varepsilon}{h^{2}}+\frac{p_{k}^{2}}{12 \varepsilon}+\frac{q_{k}}{12}-\frac{p_{k}^{\prime}}{3}\right)+\frac{h^{2} q_{k}}{72 \varepsilon}\left(\frac{p_{k}^{2}}{\varepsilon}-2 p_{k}^{\prime}-q_{k}\right)\right\} \\
& \cdot\left(w_{k-1}-w_{k}\right)+\left\{\left(\frac{\sigma_{k} \varepsilon}{h^{2}}+\frac{p_{k}^{2}}{12 \varepsilon}+\frac{q_{k}}{12}-\frac{p_{k}^{\prime}}{3}\right)\right. \\
& \left.+\frac{h^{2} q_{k}}{72 \varepsilon}\left(\frac{p_{k}^{2}}{\varepsilon}-2 p_{k}^{\prime}-q_{k}\right)\right\}\left(w_{k+1}-w_{k}\right) \\
& +\left\{\frac{p_{k}}{h}+\frac{h p_{k}\left(p_{k}^{\prime}+q_{k}\right)}{12 \varepsilon}-\frac{h p_{k}^{\prime \prime}}{6}-\frac{h q_{k}^{\prime}}{3}+\frac{h^{3} q_{k}}{72 \varepsilon}\right. \\
& \left.\cdot\left(\frac{p_{k}\left(p_{k}^{\prime}+q_{k}\right)}{\varepsilon}-p_{k}^{\prime \prime}-2 q_{k}^{\prime}\right)\right\}\left(w_{k+1}-w_{k-1}\right)+F_{k} w_{k} .
\end{aligned}
$$

For sufficiently small $h$ and for suitable values of $p_{k}$, we obtain $L^{N} w_{k}<0$. Since, $w_{k}<0$ (by assumption), $\varepsilon, \sigma_{k}>0$ and $F_{k} \longrightarrow q_{k}>0$..

However, this is a contradiction. Hence, $w_{i} \geq 0$ for all $x_{i}$ $\in(0,1)$. 
TABle 2: Maximum absolute error of Example 5 for $\varepsilon=0.1$.

\begin{tabular}{|c|c|c|c|c|}
\hline$N \longrightarrow$ & 8 & 32 & 128 & 512 \\
\hline Present method & \multicolumn{4}{|c|}{$\eta=0.5 \varepsilon$} \\
\hline \multicolumn{5}{|l|}{$\delta \downarrow$} \\
\hline 0.00 & $4.3229 e-03$ & $1.5775 e-05$ & $6.1456 e-08$ & $2.4006 e-10$ \\
\hline 0.05 & $3.8440 e-03$ & $1.3769 e-05$ & $5.4036 e-08$ & $2.1092 e-10$ \\
\hline 0.09 & $3.4760 e-03$ & $1.2460 e-05$ & $4.8494 e-08$ & $1.8940 e-10$ \\
\hline \multicolumn{5}{|l|}{ Result in [7] } \\
\hline 0.00 & 0.031377538 & 0.001800241 & 0.000112071 & $7.0036 e-06$ \\
\hline 0.05 & 0.029748010 & 0.001700026 & 0.000105418 & $6.5860 e-06$ \\
\hline 0.09 & 0.028294285 & 0.001611053 & $9.9793 e-05$ & $6.2344 e-06$ \\
\hline Present method & \multicolumn{4}{|c|}{$\delta=0.5 \varepsilon$} \\
\hline \multicolumn{5}{|l|}{$\eta \downarrow$} \\
\hline 0.00 & $3.3862 e-03$ & $1.2139 e-05$ & $4.7199 e-08$ & $1.8429 e-10$ \\
\hline 0.05 & $3.8440 e-03$ & $1.3769 e-05$ & $5.4036 e-08$ & $2.1092 e-10$ \\
\hline 0.09 & $4.2256 e-03$ & $1.5339 e-05$ & $5.9891 e-08$ & $2.3403 e-10$ \\
\hline \multicolumn{5}{|l|}{ Result in [7] } \\
\hline 0.00 & 0.027910529 & 0.001587651 & $9.8361 e-05$ & $6.1442 e-06$ \\
\hline 0.05 & 0.029748010 & 0.001700026 & 0.000105418 & $6.5860 e-06$ \\
\hline 0.09 & 0.031068500 & 0.001781207 & 0.000110800 & $6.9223 e-06$ \\
\hline
\end{tabular}

TABle 3: Maximum absolute error of Example 6 for $\varepsilon=0.1$.

\begin{tabular}{|c|c|c|c|c|}
\hline$N \longrightarrow$ & 8 & 32 & 128 & 512 \\
\hline Present method & \multicolumn{4}{|c|}{$\eta=0.5 \varepsilon$} \\
\hline \multicolumn{5}{|l|}{$\delta \downarrow$} \\
\hline 0.00 & $2.9005 e-03$ & $1.0342 e-05$ & $4.0567 e-08$ & $1.5841 e-10$ \\
\hline 0.05 & $3.5885 e-03$ & $1.2831 e-05$ & $4.9745 e-08$ & $1.9433 e-10$ \\
\hline 0.09 & $4.1815 e-03$ & $1.4979 e-05$ & $5.8027 e-08$ & $2.2664 e-10$ \\
\hline \multicolumn{5}{|l|}{ Result in [7] } \\
\hline 0.00 & 0.025347510 & 0.001425327 & $8.9204 e-05$ & $5.5742 e-06$ \\
\hline 0.05 & 0.027533826 & 0.001567710 & $9.7155 e-05$ & $6.0690 e-06$ \\
\hline 0.09 & 0.028669770 & 0.001645550 & 0.000102186 & $6.3826 e-06$ \\
\hline Present method & \multicolumn{4}{|c|}{$\delta=0.5 \varepsilon$} \\
\hline \multicolumn{5}{|l|}{$\eta \downarrow$} \\
\hline 0.00 & $1.7013 e-03$ & $1.1139 e-05$ & $4.3477 e-08$ & $1.6984 e-10$ \\
\hline 0.05 & $3.5885 e-03$ & $1.2831 e-05$ & $4.9745 e-08$ & $1.9433 e-10$ \\
\hline 0.09 & $3.9801 e-03$ & $1.4251 e-05$ & $5.5183 e-08$ & $2.1551 e-10$ \\
\hline \multicolumn{5}{|l|}{ Result in [7] } \\
\hline 0.00 & 0.026174618 & 0.001478341 & $9.2083 e-05$ & $5.7527 e-06$ \\
\hline 0.05 & 0.027533826 & 0.001567710 & $9.7155 e-05$ & $6.0690 e-06$ \\
\hline 0.09 & 0.028348272 & 0.001623113 & 0.00010057 & $6.2854 e-06$ \\
\hline
\end{tabular}


TABLe 4: Maximum absolute error of Example 7 for $\varepsilon=0.1$.

\begin{tabular}{|c|c|c|c|c|}
\hline$N \longrightarrow$ & 8 & 32 & 128 & 512 \\
\hline \multicolumn{5}{|c|}{$\eta=0.5 \varepsilon$} \\
\hline \multicolumn{5}{|l|}{$\delta \downarrow$} \\
\hline 0.00 & $9.1099 e-02$ & $1.1121 e-02$ & $6.3825 e-04$ & $4.0044 e-05$ \\
\hline 0.05 & $9.0471 e-02$ & $1.0955 e-02$ & $6.3063 e-04$ & $3.9502 e-05$ \\
\hline 0.09 & $8.9962 e-02$ & $1.0822 e-02$ & $6.2443 e-04$ & $3.9063 e-05$ \\
\hline \multicolumn{5}{|c|}{$\delta=0.5 \varepsilon$} \\
\hline \multicolumn{5}{|l|}{$\eta \downarrow$} \\
\hline 0.00 & $9.6047 e-02$ & $1.1165 e-02$ & $6.4582 e-04$ & $3.9245 e-05$ \\
\hline 0.05 & $9.6212 e-02$ & $1.1248 e-02$ & $6.4941 e-04$ & $3.9502 e-05$ \\
\hline 0.09 & $9.6342 e-02$ & $1.1313 e-02$ & $6.5223 e-04$ & $3.9705 e-05$ \\
\hline
\end{tabular}

Theorem 4. The operator $L^{N}$ in Equation (25) is stable for $\psi$ $(x)+\varphi(x)+\vartheta(x)>0$, if $w_{i}$ is any mesh function, then $\left|w_{i}\right| \leq$ $\kappa \max \left\{\left|w_{0}\right|, \max _{x_{i} \in(0,1)}\left|L w_{i}\right|\right\}$ for some constant $\kappa \geq 1$.

Proof. The proof is analogous to Theorem 2.

This proves the stability of the scheme for the case of oscillatory behaviour.

\section{Convergence Analysis}

Writing the scheme in Equation (25) in matrix form, we obtain

$$
M Y=V,
$$

where $M=\left(m_{i j}\right), i, j=1,2, \cdots, N-1$, is a tridiagonal matrix of order $N-1$. have

Multiplying both sides of Equation (25) by $\left(-h^{2}\right)$, we

$$
\begin{aligned}
m_{i i+1}= & -\sigma_{i}\left(\varepsilon-\frac{h^{2}}{6}\left(2 p_{i}^{\prime}\right)+\frac{h^{2} p_{i}^{2}}{12 \varepsilon}+\frac{h^{2} q_{i}}{12}-\frac{h^{4} q_{i}}{72 \varepsilon}\left(2 p_{i}^{\prime}+q_{i}-\frac{p_{i}^{2}}{\varepsilon}\right)\right) \\
& -\frac{h}{2}\left(p_{i}+\frac{h^{2}}{6}\left(\frac{p_{i}\left(p_{i}^{\prime}+q_{i}\right)}{\varepsilon}-p_{i}^{\prime \prime}-2 q_{i}^{\prime}\right)\right. \\
& \left.-\frac{h^{2} p_{i}\left(p_{i}^{\prime}+q_{i}\right)}{12 \varepsilon}+\frac{h^{4} q_{i}}{72 \varepsilon}\left(\frac{p_{i}\left(p_{i}^{\prime}+q_{i}\right)}{\varepsilon}-p_{i}^{\prime \prime}-2 q^{\prime}\right)\right), \\
m_{i i}=2 & \sigma_{i}\left(\varepsilon-\frac{h^{2}}{6}\left(2 p_{i}^{\prime}\right)+\frac{h^{2} p_{i}^{2}}{12 \varepsilon}+\frac{h^{2} q_{i}}{12}-\frac{h^{4} q_{i}}{72 \varepsilon}\left(2 p_{i}^{\prime}+q_{i}-\frac{p_{i}^{2}}{\varepsilon}\right)\right) \\
& -h^{2}\left(\frac{h^{2}}{6}\left(\frac{p_{i} q_{i}^{\prime}}{\varepsilon}-q_{i}^{\prime \prime}\right)-\frac{h^{2} p_{i} q_{i}}{12 \varepsilon}+q_{i}+\frac{h^{4} q_{i}}{72 \varepsilon}\left(\frac{p_{i} q_{i}^{\prime}}{\varepsilon}-q_{i}^{\prime \prime}\right)\right),
\end{aligned}
$$

$$
\begin{aligned}
m_{i i-1}= & -\sigma_{i}\left(\varepsilon-\frac{h^{2}}{6}\left(2 p^{\prime}\right)+\frac{h^{2} p_{i}^{2}}{12 \varepsilon}+\frac{h^{2} q_{i}}{12}-\frac{h^{4} q_{i}}{72 \varepsilon}\left(2 p_{i}^{\prime}+q_{i}-\frac{p_{i}^{2}}{\varepsilon}\right)\right) \\
& +\frac{h}{2}\left(p_{i}+\frac{h}{6}\left(\frac{p_{i}\left(p_{i}^{\prime}+q_{i}\right)}{\varepsilon}-p_{i}^{\prime \prime}-2 q_{i}^{\prime}\right)-\frac{h^{2} p_{i}\left(p_{i}^{\prime}+q\right)}{12 \varepsilon}\right. \\
& \left.+\frac{h^{4} q_{i}}{72 \varepsilon}\left(\frac{p_{i}\left(p_{i}^{\prime}+q_{i}\right)}{\varepsilon}-p_{i}^{\prime \prime}-2 q^{\prime}\right)\right),
\end{aligned}
$$

and $V=\left(v_{i}\right)$ is a column vector, where

$$
\begin{aligned}
v_{1} & =-h^{2}\left(D_{1}-A_{1} \alpha_{0}\right), \\
v_{i} & =-h^{2} D_{i} \quad \text { for } i=2,3, \cdots, N-2, \\
v_{N-1} & =-h^{2}\left(D_{N-1}-C_{N-1} \beta_{0}\right),
\end{aligned}
$$

with a local truncation error:

$$
T_{i}(h)=\frac{h^{6}}{15} K+O\left(h^{7}\right),
$$

where $K=\left(p_{i} / 3\right) y_{i}^{(5)}-\left(\sigma_{i} \varepsilon / 16\right) y_{i}^{(6)}$.

Equation (34) can also be written in error form as

$$
M \bar{Y}-T(h)=V
$$

where $\bar{Y}=\left(\bar{y}_{1}, \bar{y}_{2}, \cdots, \bar{y}_{N-1}\right)^{t}$ stands for the exact solution and $T(h)=\left(T_{1}(h), T_{2}(h), \cdots, T_{N-1}(h)\right)^{t}$ is a local truncation error.

From Equations (34) and (38), we obtain

$$
M(\bar{Y}-Y)=T(h)
$$

which implies

$$
M E=T(h)
$$

where $E=\bar{Y}-Y=\left(e_{1}, e_{2}, \cdots, e_{N-1}\right)^{t}$.

Let $S_{i}$ be the sum of elements of the $i^{\text {th }}$ row of a matrix $M$, then we get

$$
\begin{aligned}
& S_{i}= \sigma_{i} \varepsilon+h\left(-\frac{p_{i}}{2}\right)+h^{2}\left(-\frac{\sigma}{6}\left(2 p_{i}^{\prime}\right)+\frac{\sigma p_{i}^{2}}{12 \varepsilon}+\frac{\sigma q_{i}}{12}-q_{i}\right) \\
&+\frac{h^{3}}{6}\left(p_{i}^{\prime \prime}+2 q_{i}^{\prime}-\frac{h^{3} p_{i}\left(p_{i}^{\prime}+q_{i}\right)}{2 \varepsilon}\right)+O\left(h^{4}\right), \quad \text { for } i=1, \\
& S_{i}=h^{2}\left(-q_{i}\right)+O\left(h^{4}\right), \quad \text { for } i=2,3, \cdots, N-2,
\end{aligned}
$$


TABle 5: Numerical results of Example 7 for $\varepsilon=0.01, N=100$.

\begin{tabular}{|c|c|c|c|c|c|c|c|c|}
\hline \multirow[b]{2}{*}{$x$} & \multicolumn{2}{|c|}{$\delta=0.0, \eta=0.0$} & \multicolumn{2}{|c|}{$\delta=0.005, \eta=0.001$} & \multicolumn{2}{|c|}{$\delta=0.005=\eta$} & \multicolumn{2}{|c|}{$\delta=0.005, \eta=0.009$} \\
\hline & $\begin{array}{c}\text { Method in } \\
{[14]}\end{array}$ & $\begin{array}{l}\text { Present } \\
\text { method }\end{array}$ & $\begin{array}{c}\text { Method in } \\
{[14]}\end{array}$ & $\begin{array}{l}\text { Present } \\
\text { method }\end{array}$ & $\begin{array}{c}\text { Method in } \\
\text { [14] }\end{array}$ & $\begin{array}{l}\text { Present } \\
\text { method }\end{array}$ & $\begin{array}{c}\text { Method in } \\
\text { [14] }\end{array}$ & $\begin{array}{l}\text { Present } \\
\text { method }\end{array}$ \\
\hline 0.20 & 0.8832572 & 0.8834659 & 0.8832309 & 0.8834405 & 0.8832549 & 0.8834638 & 0.8832780 & 0.8834871 \\
\hline 0.40 & 0.7518808 & 0.7520975 & 0.7517785 & 0.7519962 & 0.7518653 & 0.7520823 & 0.7519513 & 0.7521684 \\
\hline 0.60 & 0.6265452 & 0.6266345 & 0.6263362 & 0.6264266 & 0.6265016 & 0.6265916 & 0.6266667 & 0.6267563 \\
\hline 0.80 & 0.5204743 & 0.5203749 & 0.5201598 & 0.5200613 & 0.5203944 & 0.5202959 & 0.5206292 & 0.5205302 \\
\hline 0.90 & 0.4766997 & 0.4764981 & 0.4763406 & 0.4761398 & 0.4766020 & 0.4764013 & 0.4768638 & 0.4766626 \\
\hline 0.92 & 0.4687217 & 0.4684994 & 0.4683546 & 0.4681330 & 0.4686207 & 0.4683993 & 0.4688871 & 0.4686652 \\
\hline 0.94 & 0.4610007 & 0.4607596 & 0.4606259 & 0.4603854 & 0.4608964 & 0.4606561 & 0.4611673 & 0.4609265 \\
\hline 0.96 & 0.4533263 & 0.4532756 & 0.4529480 & 0.4528939 & 0.4532202 & 0.4531689 & 0.4534931 & 0.4534435 \\
\hline
\end{tabular}

TABLE 6: Maximum absolute error for $\delta, \eta=0.5 \varepsilon$.

\begin{tabular}{|c|c|c|c|c|c|}
\hline $\begin{array}{ll}\frac{\varepsilon}{h} \\
\end{array}$ & $2^{-5}$ & $2^{-6}$ & $2^{-7}$ & $2^{-8}$ & $2^{-9}$ \\
\hline \multicolumn{6}{|c|}{ Example 5} \\
\hline $2^{-3}$ & $7.8680 e-06$ & $4.8845 e-07$ & $3.0476 e-08$ & $1.9040 e-09$ & $1.1899 e-10$ \\
\hline $2^{-4}$ & $4.8096 e-05$ & $2.9675 e-06$ & $1.8546 e-07$ & $1.1578 e-08$ & $7.2378 e-10$ \\
\hline $2^{-5}$ & $3.4554 e-04$ & $1.9965 e-05$ & $1.2232 e-06$ & $7.6501 e-08$ & $4.7753 e-09$ \\
\hline $2^{-6}$ & $2.1829 e-03$ & $1.5228 e-04$ & $8.8556 e-06$ & $5.4340 e-07$ & $3.3824 e-08$ \\
\hline $2^{-7}$ & $9.4645 e-03$ & $1.0205 e-03$ & $7.0761 e-05$ & $4.1300 e-06$ & $2.5365 e-07$ \\
\hline $2^{-8}$ & $2.8510 e-02$ & $4.4918 e-03$ & $4.9169 e-04$ & $3.4000 e-05$ & $1.9883 e-06$ \\
\hline \multicolumn{6}{|c|}{ Example 6} \\
\hline $2^{-3}$ & $7.8654 e-06$ & $8.8695 e-07$ & $3.0790 e-08$ & $3.4972 e-09$ & $1.2029 e-10$ \\
\hline $2^{-4}$ & $3.6536 e-05$ & $2.2720 e-06$ & $1.4204 e-07$ & $8.8711 e-09$ & $5.5450 e-10$ \\
\hline $2^{-5}$ & $1.8558 e-04$ & $1.1830 e-05$ & $7.3360 e-07$ & $4.5832 e-08$ & $2.8636 e-09$ \\
\hline $2^{-6}$ & $1.2043 e-03$ & $6.8432 e-05$ & $4.2579 e-06$ & $2.6466 e-07$ & $1.6561 e-08$ \\
\hline $2^{-7}$ & $6.7569 e-03$ & $4.7969 e-04$ & $2.7698 e-05$ & $1.6967 e-06$ & $1.0622 e-07$ \\
\hline $2^{-8}$ & $2.8776 e-02$ & $2.9911 e-03$ & $2.0743 e-04$ & $1.2083 e-05$ & $7.4178 e-07$ \\
\hline \multicolumn{6}{|c|}{ Example 7} \\
\hline $2^{-3}$ & $8.3545 e-03$ & $2.0138 e-03$ & $4.9861 e-04$ & $1.2495 e-04$ & $3.1217 e-05$ \\
\hline $2^{-4}$ & $1.7199 e-02$ & $4.3785 e-03$ & $1.0417 e-03$ & $2.5713 e-04$ & $6.4294 e-05$ \\
\hline $2^{-5}$ & $2.5179 e-02$ & $8.8894 e-03$ & $2.2383 e-03$ & $5.2902 e-04$ & $1.3037 e-04$ \\
\hline $2^{-6}$ & $3.1540 e-02$ & $1.2943 e-02$ & $4.5167 e-03$ & $1.1313 e-03$ & $2.6648 e-04$ \\
\hline $2^{-7}$ & $4.4783 e-02$ & $1.6224 e-02$ & $6.5594 e-03$ & $2.2763 e-03$ & $5.6865 e-04$ \\
\hline $2^{-8}$ & $7.8783 e-02$ & $2.3176 e-02$ & $8.2240 e-03$ & $3.3015 e-03$ & $1.1426 e-03$ \\
\hline
\end{tabular}




$$
\begin{aligned}
S_{i}= & \sigma_{i} \varepsilon+h\left(\frac{p_{i}}{2}\right)+h^{2}\left\{-\frac{\sigma}{6}\left(2 p_{i}^{\prime}\right)+\frac{\sigma p_{i}^{2}}{12 \varepsilon}+\frac{\sigma q_{i}}{12}+\frac{1}{12}\right. \\
& \left.\cdot\left(\frac{p_{i}\left(p_{i}^{\prime}+q_{i}\right)}{\varepsilon}-p_{i}^{\prime \prime}-2 q_{i}^{\prime}\right)-q_{i}\right\}+h^{3}\left(-\frac{p_{i}\left(p_{i}^{\prime}+q_{i}\right)}{24 \varepsilon}\right) \\
& +O\left(h^{4}\right), \quad \text { for } i=N-1 .
\end{aligned}
$$

For a sufficiently small $h$, the matrix $M$ is irreducible and monotone [1]. Thus, $M^{-1}$ exists and $M^{-1} \geq 0$.

Thus, Equation (40), gives

$$
\begin{gathered}
E=M^{-1} T(h), \\
\|E\| \leq\left\|M^{-1}\right\| \cdot\|T(h)\| .
\end{gathered}
$$

Let $\bar{m}_{k, i} \geq 0$ be the $(k, i)^{\text {th }}$ element of $M^{-1}$. From the theory of matrices, we have

$$
\sum_{i=1}^{N-1} \bar{m}_{k, i} S_{i}=1, \quad k=1,2, \cdots, N-1 .
$$

Therefore,

$$
\sum_{i=1}^{N-1} \bar{m}_{k, i} \leq \frac{1}{\min _{1 \leq i \leq N-1} S_{i}}=\frac{1}{h^{2}\left|B_{i_{0}}\right|},
$$

where $B_{i_{0}}=-q_{i}$.

We define $\left\|M^{-1}\right\|=\max _{1 \leq k \leq N-1} \sum_{i=1}^{N-1}\left|\bar{m}_{k, i}\right|$ and $\|T(h)\|=$ $\max _{1 \leq i \leq N-1}\left|T_{i}(h)\right|$.

From Equations (34), (42), (43), and (45), we get

$$
e_{i}=\sum_{k=1}^{N-1} \bar{m}_{k, i} T_{i}(h), \quad i=1,2, \cdots, N-1,
$$

which implies

$$
e_{i} \leq\left(\sum_{k=1}^{N-1} \bar{m}_{k, i}\right) \max _{1 \leq i \leq N-1}\left|T_{i}(h)\right| \leq \frac{h^{6} K}{15 h^{2}\left|B_{i_{0}}\right|}=\frac{h^{4} K}{15\left|B_{i_{0}}\right|},
$$

where $i_{0}$ is some number between $i$ and $N$.

Therefore, $\|E\|=O\left(h^{4}\right)$. Hence, the present method is of fourth-order convergence.

\section{Numerical Examples and Results}

To show the applicability of the method, three model examples have been considered. The exact solution of Equations (1) and (2) with constant coefficients is

$$
y(x)=c_{1} e^{m_{1} x}+c_{2} e^{m_{2} x}+\frac{r}{c_{3}},
$$

where

$$
\begin{gathered}
m_{1}=\frac{\left\{\delta \psi-\phi-\eta \vartheta+\operatorname{sqrt}\left((\delta \psi-\phi-\eta \vartheta)^{2}-4 \varepsilon c_{3}\right)\right\}}{2 \varepsilon}, \\
m_{2}=\frac{\left\{\delta \psi-\phi-\eta \vartheta-\operatorname{sqrt}\left((\delta \psi-\phi-\eta \vartheta)^{2}-4 \varepsilon c_{3}\right)\right\}}{2 \varepsilon}, \\
c_{3}=\psi+\varphi+\vartheta, \\
c_{1}=\frac{\left(-r+\beta c_{3}+e^{m_{2}}\left(r-\alpha c_{3}\right)\right)}{c_{3}\left(e^{m_{1}}-e^{m_{2}}\right)}, c_{2}=\frac{\left(r-\beta c_{3}+e^{m_{1}}\left(-r+\alpha c_{3}\right)\right)}{c_{3}\left(e^{m_{1}}-e^{m_{2}}\right)} .
\end{gathered}
$$

For the variable coefficients, the maximum absolute errors are computed using the double mesh principle [13].

Example 5. Consider the model equation (7),

$$
\varepsilon y^{\prime \prime}(x)-y^{\prime}(x)-2 y(x-\delta)+y(x)-2 y(x+\eta)=0,
$$

with boundary conditions,

$$
y(x)=1,-\delta \leq x \leq 0, y(1)=-1,1 \leq x \leq \eta .
$$

Example 6. Consider the model equation (7),

$$
\varepsilon y^{\prime \prime}(x)+0.5 y^{\prime}(x)-3 y(x-\delta)-2 y(x)+2 y(x+\eta)=1,
$$

with boundary conditions,

$$
y(x)=1,-\delta \leq x \leq 0, y(1)=0,1 \leq x \leq \eta .
$$

Example 7. Consider the model equation (14),

$$
\varepsilon y^{\prime \prime}(x)-\left(1+e^{x^{2}}\right) y^{\prime}(x)-x y(x-\delta)+x^{2} y(x)-\left(1-e^{-x}\right) y(x+\eta)=1,
$$

with boundary conditions,

$$
y(x)=1,-\delta \leq x \leq 0, y(1)=-1,1 \leq x \leq \eta .
$$

The following graphs (Figures 1 and 2) show the effect of delay and advance parameters on the solution profile.

The following graphs (Figure 3) show the pointwise absolute errors for different values of mesh size $h$.

\section{Discussion and Conclusion}

This study introduces an exponentially fitted fourth-order numerical method for solving singularly perturbed differential-difference equations. The results observed from the tables demonstrate that the present method approximates the solution very well and depicts the betterment over some existing numerical methods reported in the literature. The stability and convergence of the scheme have been established. The solutions presented in Table 1 confirm that the numerical rate of convergence as well as theoretical error 
estimates indicates that the present method is of fourth-order convergence.

To demonstrate the effect of delay and advance parameters on the left and right boundary layers of the solution, the graphs for different values of delay parameter $\delta$ and advance parameter $\eta$ are plotted in Figures 1 and 2. Accordingly, based on the sign of $p(x)$, one can see that, from Figure 1, as $\delta$ increases, the width of the right boundary layer decreases for a fixed value of $\eta$, but as $\eta$ increases, the width of the right boundary layer increases for a fixed value of $\delta$ while the width of the left boundary layer decreases when $\delta$ or $\eta$ increases (Figure 2). Furthermore, as $h$ decreases, the absolute error also decreases (see Tables 2-6 and Figure 3). In general, the present method is stable, convergent, and more accurate.

\section{Nomenclature}

$\begin{array}{ll}\varepsilon: & \begin{array}{l}\text { Perturbation } \\ \text { parameter }\end{array} \\ \delta: & \text { Delay } \\ & \text { parameter } \\ \eta: & \text { Advance } \\ & \text { parameter } \\ \sigma: & \text { Fitting } \\ & \text { parameter. } \\ \phi(x), \psi(x), \varphi(x), \vartheta(x), r(x), \alpha(x), \text { and } \beta(x): & \begin{array}{l}\text { Smooth } \\ \text { functions. }\end{array}\end{array}$

\section{Data Availability}

No data were used to support this study.

\section{Conflicts of Interest}

The authors declare that they have no conflicts of interest.

\section{Acknowledgments}

The authors wish to express their thanks to Jimma University and Madda Walabu University for material support.

\section{References}

[1] G. File, G. Gadisa, T. Aga, and Y. N. Reddy, "Numerical solution of singularly perturbed delay reaction-diffusion equations with layer or oscillatory behavior," American Journal of Numerical Analysis, vol. 5, no. 1, pp. 1-10, 2017.

[2] K. Hosseinzadeh, A. Asadi, A. R. Mogharrebi, M. Ermia Azari, and D. D. Ganji, "Investigation of mixture fluid suspended by hybrid nanoparticles over vertical cylinder by considering shape factor effect," Journal of Thermal Analysis and Calorimetry, vol. 143, no. 2, pp. 1081-1095, 2021.

[3] K. Hosseinzadeh, S. Roghani, A. R. Mogharrebi, A. Asadi, M. Waqas, and D. D. Ganji, "Investigation of cross-fluid flow containing motile gyrotactic microorganisms and nanoparticles over a three-dimensional cylinder," Alexandria Engineering Journal, vol. 59, no. 5, pp. 3297-3307, 2020.

[4] A. R. Mogharrebi, A. R. D. Ganji, K. Hosseinzadeh, S. Roghani, A. Asadi, and A. Fazlollahtabar, "Investigation of magnetohy- drodynamic nanofluid flow contain motile oxytactic microorganisms over rotating cone," International Journal of Numerical Methods for Heat \& Fluid Flow, 2021.

[5] K. Hosseinzadeh, E. Montazer, M. B. Shafii, and D. D. Ganji, "Heat transfer hybrid nanofluid (1-butanol/ $\mathrm{MoS}_{2}-\mathrm{Fe}_{3} \mathrm{O}_{4}$ ) through a wavy porous cavity and its optimization," International Journal of Numerical Methods for Heat \& Fluid Flow, vol. 31, no. 5, pp. 1547-1567, 2021.

[6] K. Phaneendra, G. B. S. L. Soujanya, and Y. N. Reddy, "Numerical solution of second order singularly perturbed differentialdifference equation with negative shift," International Journal of Nonlinear Science, vol. 18, no. 3, pp. 200-209, 2014.

[7] D. Kumara Swamy, K. Phaneendra, and Y. N. Reddy, "Solution of singularly perturbed differential-difference equations with mixed shifts using Galerkin method with exponential fitting," Chinese Journal of Mathematics, vol. 2016, Article ID 1935853, 10 pages, 2016.

[8] R. Kanth and M. M. Kumar, "Numerical treatment for a singularly perturbed convection delayed dominated diffusion equation via tension splines," International Journal of Pure and Applied Mathematics, vol. 113, no. 6, pp. 1314-3395, 2017.

[9] G. File, A. Andargie, and Y. N. Reddy, “A fitted-stable central difference method is solving singularly perturbed two point boundary value problems with the boundary layer at one end (left or right) of the interval," Ethiopian Journal of Education and Sciences, vol. 11, no. 1, pp. 61-76, 2015.

[10] M. K. Kadalbajoo and V. P. Ramesh, "Hybrid method for numerical solution of singularly perturbed delay differential equations," Applied Mathematics and Computation, vol. 187, no. 2, pp. 797-814, 2007.

[11] M. K. Kadalbajoo and D. Kumar, "Fitted mesh B-spline collocation method for singularly perturbed differential-difference equations with small delay," Applied Mathematics and Computation, vol. 204, no. 1, pp. 90-98, 2008.

[12] L. Sirisha and Y. N. Reddy, "Solution of singularly perturbed differential-difference equations using higher order finite differences," American Journal of Numerical Analysis, vol. 3, no. 1, pp. 8-17, 2015.

[13] G. Gadisa, G. File, and T. Aga, "Fourth order numerical method for singularly perturbed delay differential equations," International Journal of Applied science and Engineering, vol. 15, no. 1, pp. 16-31, 2018.

[14] G. File and Y. N. Reddy, "Fitted-modified upwind finite difference method for solving singularly perturbed differential difference equations," International Journal of Mathematical Models and Methods in Applied Sciences, vol. 6, no. 7, pp. 791-802, 2012.

[15] P. Mushahary, S. R. Sahu, and J. Mohapatra, "A parameter uniform numerical scheme for singularly perturbed differentialdifference equations with mixed shifts," Journal of Applied and Computational Mechanics, vol. 6, no. 2, pp. 344-356, 2020.

[16] L. E. Elsgolt's and S. B. Norkin, Introduction to the Theory and Application of Differential Equations with Deviating Arguments, Academic Press, New York, 1973.

[17] R. E. O’Malley, Introduction to Singular Perturbations, Academic Press, New York, 1974. 